\title{
用 $\alpha$-甲氧基苯乙酸为鋯的沉淀剂
}

\author{
梁树权王順荣
}

(中国科学院化学研究所)

苦杏仁酸 ( $\alpha$-羥基苯基乙酸) 是鋯的专一試剂。 鋯与苦杏仁酸的羧基以电价結合，与垟基的氧共价 結合 ${ }^{[1]}$ 。因此，将苦杏仁酸的羥基变为甲氧基是否 仍能沉淀鉛是一个有兴趣的問題。試驗結果找出 $\boldsymbol{\alpha}$-甲氧基苯基乙酸能从含数克氯 化銨 的 $\mathrm{pH} 0.8$ 1.4 的鋯溶液中析出白色粉末状的沉 淀。經 分析, 得知此沉淀中鋯和有机部分的克分子比为 $1: 1$, 故 此沉淀应系碱式 $\alpha$-甲氧基苯基乙酸鋯。按苦杏仁 酸鋯能从强酸介稹(高至 $5 N$ 盐酸) 中沉淀及能溶于 氢氯化銨，而此沉淀缺乏上述性盾。由此可見苦杏 仁酸之 $\alpha$-垹基經甲基化后，影响其氯的配位能力。

定量沉淀需加入理諭量 (以 $1: 1$ 計算) 十六倍的 沉淀剂。沉淀后最好在水浴上放置两小时, 以促使 定量沉腚。所得沉腚用致密滤紙过滤， $0.1 \%$ 沉淀 剂的 $0.1 N$ 盐酸溶液洗桬，及最后灼烧为二氯化鋯
至唒重。用此試剂可定量沉淀少至 2.6 毫克鋯。67

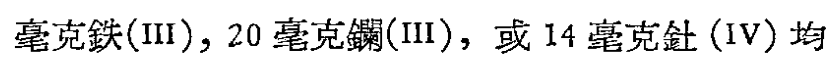
不干扰。但 2 毫克鈦(IV)或 10 毫克以上的硫酸根 干扰。

碱式 $\alpha$-甲氧基苯基乙酸鋯的热分解曲綫 不似 苦杏仁酸鉛 ${ }^{[2]}$ ，后者有两平台（在 $60-188^{\circ} \mathrm{C}$ 和 $820-960^{\circ} \mathrm{C}$ ) 而前者仅有一平台在 $815-910^{\circ} \mathrm{C}$ (以 上未試驗)。因此本沉淀只能灼烧为二氧化鋯 而 不 能以碱式盐衡重。

[1] Feigl F., "Chemistry of Specific, Selective and Sensitive Reaetions", p. 213, Academic Press, New York, 1949;

Stromatt R. W., Ph. D. Thesis, Kansas State Univ., 1958; Dissertation Abs., 18, 1609 (1958).

[2] Duval Cl., "Inorganic Thermogravimetric Analysis", pp. 323-324, Elsevier, Amsterdam, 1953.

\section{$\mathrm{V}_{2} \mathrm{O}_{5}$-体系催化剂的电子磁共振譜}

\author{
徐广智陈素明毕”视唐有稘
}

(中国科学院化学研究所)

$\mathrm{V}_{2} \mathrm{O}_{5}$ 作为催化剂极其普遍地用于 $\mathrm{SO}_{2}$ 氯化为 $\mathrm{SO}_{3}$ 和有机物的氧化反应中。为提高其选择性和催 化活性，往往与其他輔助催化剂如 $\mathrm{K}_{2} \mathrm{SO}_{4} 、 \mathrm{~K}_{2} \mathrm{~S}_{2} \mathrm{O}_{7}$ 、 $\mathrm{MoO}_{3} 、 \mathrm{WO}_{3}$ 及 $\mathrm{P}_{2} \mathrm{O}_{5}$ 等合抹使用。最近 B. 6. 卡 贊斯基 ${ }^{[1,2]}$ 等測得了某些 $\mathrm{V}_{2} \mathrm{O}_{5}$ 体采催化剂的电子 磁共振譜, 作者 ${ }^{[3]}$ 等継研究 $\mathrm{V}_{2} \mathrm{O}_{5}-\mathrm{K}_{2} \mathrm{~S}_{2} \mathrm{O}_{8}$ 体系电
子磁共振譜之后, 及探討了 $\mathrm{V}_{2} \mathrm{O}_{5}-\mathrm{K}_{2} \mathrm{SO}_{4} 、 \mathrm{~V}_{2} \mathrm{O}_{5}$ 一 $\mathrm{KHSO}_{4}$ 及 $\mathrm{V}_{2} \mathrm{O}_{5}-\mathrm{K}_{2} \mathrm{~S}_{2} \mathrm{O}_{7}$ 等体系的磁共振譜。

实驗部分:

1.样品制备：

取化学純的 $\mathrm{V}_{2} \mathrm{O}_{5}$ 分別与 $\mathrm{K}_{2} \mathrm{SO}_{4} 、 \mathrm{KHSO}_{4}$ 及 $\mathrm{K}_{2} \mathrm{~S}_{2} \mathrm{O}_{7}$ 按表 1-3 中的比例混合均匀后, 于 $500{ }^{\circ} \mathrm{C}$ 
加热牛小时或一小时，得深色产物。

2. 样品中四价釚的測定:

溶解适量样品于 $2 \mathrm{~N} \mathrm{H}_{2} \mathrm{SO}_{4}$ 溶液中，以标准高 錳酸鉀溶液滴定，由其消耗量算出的样品中四价釩 含量見表 1 - 3 。

表 1

\begin{tabular}{c|c|c|c}
\hline 样 品 & $\mathrm{V}_{2} \mathrm{O}_{5} \%$ (摩尔) & $\begin{array}{c}\mathrm{KHSO}_{4} \% \\
(\text { 摩尔) }\end{array}$ & $\begin{array}{c}\mathrm{V}^{+4} \% \\
\text { (重量) }\end{array}$ \\
\cline { 1 - 2 } 1 & 2.6 & 97.4 & 0.11 \\
2 & 5.3 & 94.7 & 0.30 \\
3 & 8.1 & 91.9 & 0.64 \\
4 & 11.0 & 89.0 & 0.88 \\
5 & 14.3 & 85.7 & 1.17 \\
6 & 17.7 & 82.3 & 2.03 \\
7 & 25.0 & 75.0 & 2.91 \\
8 & 33.3 & 67.7 & 2.66 \\
\hline
\end{tabular}

\begin{tabular}{c|c|c|c}
\multicolumn{3}{|c|}{ 表 2} \\
\hline 样 品 & $\mathrm{V}_{2} \mathrm{O}_{5} \%$ (摩尔) & $\begin{array}{c}\mathrm{K}_{2} \mathrm{SO}_{4} \% \\
\text { (摩尔) }\end{array}$ & $\mathrm{V}^{+4} \%$ (重量) \\
\hline 1 & 1 & 99 & 0.03 \\
2 & 3 & 97 & 0.05 \\
3 & 5 & 95 & 0.06 \\
4 & 10 & 90 & 0.10 \\
5 & 15 & 85 & 0.17 \\
6 & 20 & 80 & 0.32 \\
7 & 25 & 75 & 0.32 \\
8 & 30 & 70 & 0.34 \\
9 & 40 & 60 & 0.49 \\
10 & 50 & 50 & 0.53 \\
\hline
\end{tabular}

表 3

\begin{tabular}{c|c|c|c}
\hline 样 品 & $\mathrm{V}_{2} \mathrm{O}_{5} \%$ (摩尔) & $\begin{array}{c}\mathrm{K}_{2} \mathrm{~S}_{8} \mathrm{O}_{7} \% \\
\text { (摩尔) }\end{array}$ & $\mathrm{V}^{+4} \%$ (重量) \\
\hline 1 & 5 & 95 & 0.14 \\
2 & 10 & 90 & 0.27 \\
3 & 15 & 85 & 0.54 \\
4 & 20 & 80 & 0.81 \\
5 & 25 & 75 & 1.18 \\
6 & 30 & 70 & 1.62 \\
7 & 40 & 60 & 2.62 \\
8 & 50 & 50 & 2.26 \\
\hline
\end{tabular}

3. 电子磁共振譜的測定:

于波长为 3.2 公分的高頻調場波譜仪中，在室
溫下測得了 $\mathrm{V}_{2} \mathrm{O}_{5}-\mathrm{K}_{2} \mathrm{SO}_{4} 、 \mathrm{~V}_{2} \mathrm{O}_{5}-\mathrm{KHSO}_{4}$ 及 $\mathrm{V}_{2} \mathrm{O}_{5}-$ $\mathrm{K}_{2} \mathrm{~S}_{2} \mathrm{O}_{7}$ 等体系所有样品的电子磁共振譜, 而 $\mathrm{V}_{2} \mathrm{O}_{5}$ 、 $\mathrm{K}_{2} \mathrm{SO}_{4} 、 \mathrm{~K}_{2} \mathrm{~S}_{2} \mathrm{O}_{7}$ ，和在 $500{ }^{\circ} \mathrm{C}$ 加热后的 $\mathrm{K}_{2} \mathrm{SO}_{4}$ 、 $\mathrm{KHSO}_{4}$ 及 $\mathrm{K}_{2} \mathrm{~S}_{2} \mathrm{O}_{7}$. 却不呈現任何共振信号。

\section{4.实驗結果及討論:}

根据 $\mathrm{K}_{2} \mathrm{SO}_{4} 、 \mathrm{KHSO}_{4}$ 及 $\mathrm{K}_{2} \mathrm{~S}_{2} \mathrm{O}_{7}$ 无共振吸收， 而在 $500^{\circ} \mathrm{C}$ 加热后的 $\mathrm{V}_{2} \mathrm{O}_{5}$ 却有共振信号的事实, 和釚离子的电子組态, 可知 $\mathrm{V}_{2} \mathrm{O}_{5}-\mathrm{K}_{2} \mathrm{SO}_{4} 、 \mathrm{~V}_{2} \mathrm{O}_{5}-$ $\mathrm{KHSO}_{4}$ 及 $\mathrm{V}_{2} \mathrm{O}_{5}-\mathrm{K}_{2} \mathrm{~S}_{2} \mathrm{O}_{7}$ 等体系的电子磁共振譜只 能是低价猃离子中未偶电子的标志, 但在高溫及非 惰性气氛的条件下，低价釚离子 $\mathrm{v}^{+2} 、 \mathrm{v}^{+3}$ 等存在 的可能性极小，因此可以訩为此等体系的共振譜是 由四价釚离子貢献的。

在 $\mathrm{V}_{2} \mathrm{O}_{5}-\mathrm{K}_{2} \mathrm{~S}_{2} \mathrm{O}_{7}$ 体系中， $\mathrm{V}_{2} \mathrm{O}_{5}$ 含量等于或 低于 $30 \%$ (摩尔)时, 其共振譜具有分辩得很清楚的 十三重精細結构; 当 $\mathrm{V}_{2} \mathrm{O}_{5}$ 含量堆至 $50 \%$ (摩尔)时 共振謭变为一寬綫，如图 1 所示。

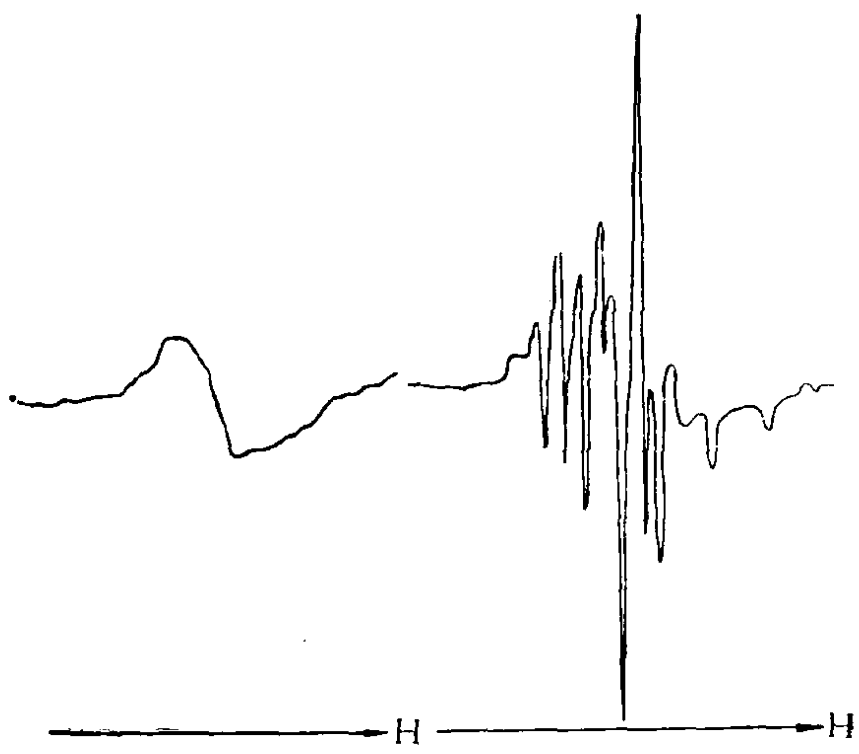

图 1-a

图 1-b

$50 \% \mathrm{~V}_{8} \mathrm{O}_{5}$ $30 \% \mathrm{~V}_{2} \mathrm{O}_{5}$

在 $\mathrm{V}_{2} \mathrm{O}_{5}$ 一 $\mathrm{KHSO}_{4}$ 体系中, $\mathrm{V}_{2} \mathrm{O}_{5}$ 含量低于 $17.7 \%$ (摩尔)时, 其共振謭也有很清楚的精細結构, 抹与 $\mathrm{V}_{2} \mathrm{O}_{5}-\mathrm{K}_{2} \mathrm{~S}_{2} \mathrm{O}_{7}$ 体系的相同; 超过 $17.7 \%$ (摩尔)时， 共振譜的精細結构郎模糊不清。如果考虑反应

$$
2 \mathrm{KHSO}_{4}=\mathrm{K}_{2} \mathrm{~S}_{2} \mathrm{O}_{7}+\mathrm{H}_{2} \mathrm{O}
$$

的存在, 則 $\mathrm{V}_{2} \mathrm{O}_{5}-\mathrm{KHSO}_{4}$ 体采中 $\mathrm{V}_{2} \mathrm{O}_{5}$ 含量为 $17.7 \%$ (縻尔)的样品就相当于 $\mathrm{V}_{2} \mathrm{O}_{5}-\mathrm{K}_{2} \mathrm{~S}_{2} \mathrm{O}_{7}$ 体系 


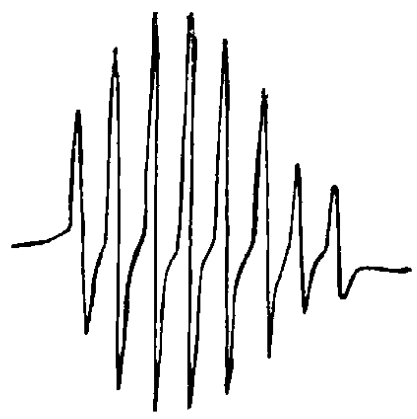

H

图 2-a $\mathrm{VO}^{++}$与乙酰丙酮的絡合物在 氯仿中的共振譜

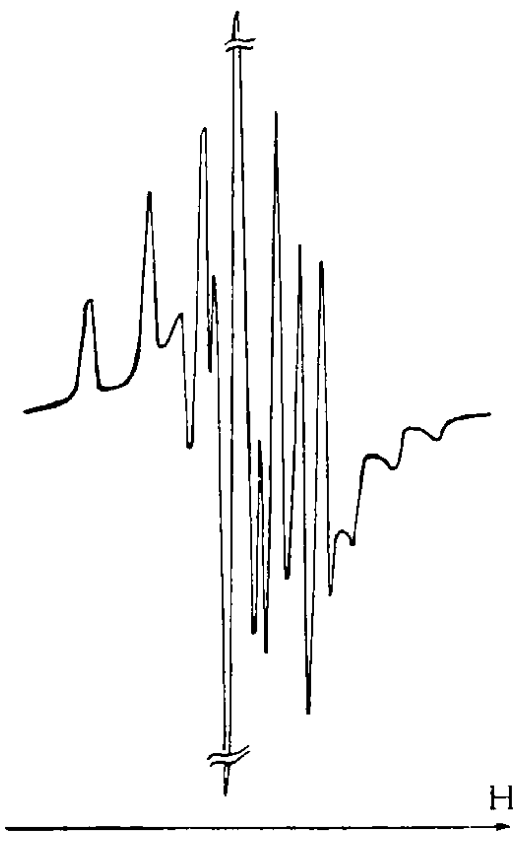

图 2-b 吸附在 $\mathrm{SiO}_{2}-\mathrm{Al}_{2} \mathrm{O}_{8}$ 表面上的 $\mathrm{VO}^{++}$与 乙酸丙酮的絡合物的电子磁共振譜

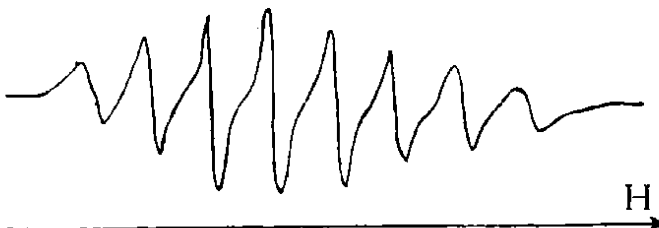

图 2-c VO+H 与吓啉的絡合物在苯中的共振譜

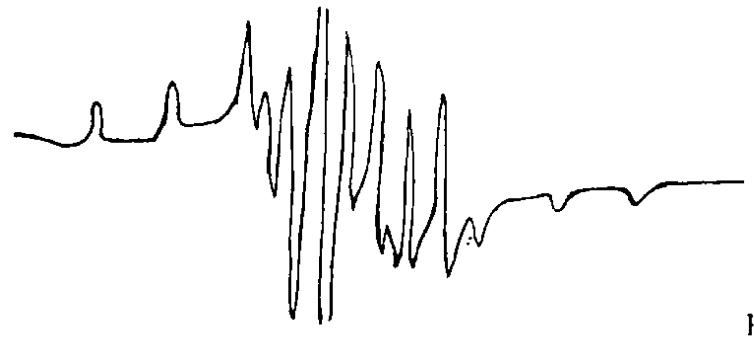

图 2-d $\mathrm{V}^{++}$与吓啉的絡合物在炶度大的油中的 共振譜
中 $\mathrm{V}_{2} \mathrm{O}_{5}$ 含量为 $30 \%$ (摩尔) 的样品, 因此二者共振 譄精細結构随 $\mathrm{V}_{2} \mathrm{O}_{5}$ 含量变化的对应情况正是所預 期的。

$\mathrm{V}_{2} \mathrm{O}_{3}-\mathrm{K}_{2} \mathrm{SO}_{4}$ 体系中，当 $\mathrm{V}_{2} \mathrm{O}_{5}$ 含量超过 $20 \%$ (縻尔)时, 其共振熋有較为清楚的精細結构出現, 綫形与 $\mathrm{V}_{2} \mathrm{O}_{5}-\mathrm{KHSO}_{4}$ 及 $\mathrm{V}_{2} \mathrm{O}_{5}-\mathrm{K}_{2} \mathrm{~S}_{2} \mathrm{O}_{7}$ 体系的相 似。但在 $\mathrm{V}_{2} \mathrm{O}_{5}$ 含量低于 $20 \%$ (摩尔)时, 共振譜的 精細結构不甚明显。由表 2 中的数据可知, 这不太 可能是由于其中四价钥含量太低，使共振信号太弱 而引起的結果，而是超过 $20 \%$ 时，体栾变为玻琌 状，使 $\mathrm{V}^{+4}$ 間距离增大所致。

比較 $\mathrm{V}_{2} \mathrm{O}_{5}-\mathrm{K}_{2} \mathrm{SO}_{4} 、 \mathrm{~V}_{2} \mathrm{O}_{5}$ 一 $\mathrm{KHSO}_{4}$ 及 $\mathrm{V}_{2} \mathrm{O}_{5}-$ $\mathrm{K}_{2} \mathrm{~S}_{2} \mathrm{O}_{7}$ 等三体采共振譜精細結构的数目和綫形，显 然可以設想它們的共振諧是由类同的四价釷离子所 产生的。在 $\mathrm{V}_{2} \mathrm{O}_{5}-\mathrm{KHSO}_{4} 、 \mathrm{~V}_{2} \mathrm{O}_{5}-\mathrm{K}_{2} \mathrm{~S}_{2} \mathrm{O}_{7}$ 体系中 $\mathrm{V}_{2} \mathrm{O}_{5}$ 含量增加时，四价釚浓度也相应增加，其間 距減少，偶极一偶极作用会使譜綫增寬，由表 I、

表 3 可胃虽然四价猃含量在 $\mathrm{V}_{2} \mathrm{O}_{5}$ 含量增加到一定 程度时反而会減少，但釚核总数的增多，也有类似 的增寬效果, 故当 $\mathrm{V}_{2} \mathrm{O}_{5}$ 含量超过一定量时, 共振 譄就变为一寬綫。

为了肯定体系中四价钥存在的形式，作者仿 Faber ${ }^{[4]}$ 等人的方法，測定了昅附在离子交換 树脂 上的 $\mathrm{vO}^{++}$离子的共振措, 其精細結构数目 和 綫 形与图 1 中的类同，因此可訩为体系中四价唋是以 $\mathrm{vO}^{++}$的形式存在。同时釩有本度为 $99.75 \%$ 的同 位素之原子核自旋 $I=7 / 2$ ，因而未偶电子与原子 核磁矩間的相互作用，应产生八重吸收峯，如果再 考虑到体系中順磁离子处晶体場对称軸对外磁場取 向的机遇分布，按 Sands ${ }^{[5]}$ 的理論应得两組八重吸 收崟，但其中部分較弱或有所重迭，故得如实測的 十三重吸收峯。为了証明这一推断，作者測定了 $\mathrm{VO}^{++}$与乙酰丙酮的絡合物在氮仿中及其被吸附 在 $\mathrm{SiO}_{2}-\mathrm{Al}_{2} \mathrm{O}_{3}$ 催化剂表面后的共振譜（如图 3-a、 3-b 所示), 抹和 Roberts[7,8] 等得到的 vo ${ }^{++}$与叶 啉的絡合物在苯中及粘度大的油中的共振譩（如 图 3-c、3-d）以及本交所得譜进行了比較。两种絡合 物在粘度小的溶液中分子轉动甚快，表現出超精細 分裂是各向同性的，所以都具八重綫，但 $\mathrm{VO}^{++}$与 
吓啉的絡合物在粘度大的油中，分子轉动葚慢，其 晶体場对称軸对外磁場取向是机遇分布的，而吸附 在 $\mathrm{SiO}_{2}-\mathrm{Al}_{2} \mathrm{O}_{3}$ 表面上的 $\mathrm{VO}^{++}$与乙酰丙酩的絡合 物中, 順磁离子处晶体場对称軸也是机遇取向的, 得相同的十三重吸收峯，抹且它們与图 1 的譜完全 一样，从而佐証了上述推断。

[1] Қазанский В. Б., Ежкова 3. И., ЛюБарекий А. Г., Воеводский В. В. и Иоффе И. И.,
Кинетика и катализ, 2, 862 (1961).

[2 ] Tarama K., Teranishi S., Yoshida S. and Yoshida H., Bull. Chem. Soc. Japan, 34, 1195 (1961).

[3] 徐广智、唐有掑, 科学通报, 1963 年, 1 月号, 53頁。

[4] Faber R. J. and Rogers M. T., J. Amer. Chem. Soc., 81, 1849 (1959).

[ 5 ] Sands R. H., Phys. Rer., 99, 1222 (1955).

[6]徐广智、陈素明、李立捲、唐有䄍, 待发表。

[ 7 ] Roberts E. M., Koski W. S. and Caughey W. S., J. Chem. Phys., 34, 591 (1961).

[8] O'Reilly D. E., J. Chem. Phys., 29, 1188 (1959).

\section{芳烃負离子基的順磁共振研究}

\section{I. 三联苯異构体貰离子基}

朱自犖傅桂香潘家来盛怀禹胡裕杰

（中国科学院有机化学㸴究所）

有关芳炡离子基的順磁共振謭研究，近年来取 代苯 ${ }^{[1]}$ 及稠环二种筫离壬基的报导較多，而联苯系 負离子基研究較少。其中二联苯負离子基曾由九綫 譜 ${ }^{[3]}$ 扩展为九組五綫譜 ${ }^{[4] *}$ ，而对 三联苯負离子基 由单綫 ${ }^{[3]}$ 扩展为八綫 ${ }^{[5]}$ 。在上述諸报导中对所得譜 图均未曾作出任何理諭的說明。我們會获得高浓度 对一三联苯負樆子基溶液的諧图为单綫 及九綫，而 当稀释到适当的浓度时,譄綫数目达到 35 綫。至于 邻一攻間-三联苯負离子基的电子順磁共振譜迄今向 未見諸报导，所以联苯系負离子基的电子順磁共振 研究是极不完整的。为此我們合成了三联苯更四联 苯各异构体，以便总結联苯系負离子基的順磁共振 譄的規律，抹探討其結构問題。

本文主要报导三联苯各异构体的順磁共振譩， 以后还发表四联苯异构体的譜图。邻一及对-三联苯 負离子基均为 35 綫，而閒-三联苯为 17 組 5 綫。从 已有的材料来看，对-三联苯負离子基各碳原子上 的自旋密度值服从 Hückel 分子軌道法計算結果。
在三苯环之間未偶电子均可传递。

\section{实 驗 部 分}

一、样品制备 ${ }^{[6]}$ :邻一三联苯以 Wurtz 法制备。 間-三联苯系以邻-三联苯經无水三氯化鋁之催化异 构制得，而对三联苯系以間三联苯經高溫催化异构 获得。上述三种产物均經分餾及重結晶数次純化。 純样品的紫外光譜**与文献相符 ${ }^{[7]}$ 。各三联苯异构 体的物化数据为: 邻-三联苯的沸点 $331^{\circ} \mathrm{C}$ 及熔点 $58-59^{\circ} \mathrm{C}$; 間-三联苯的沸点 $368^{\circ} \mathrm{C}$ 及熔点 $88^{\circ} \mathrm{C}$; 对-三联苯的沸点 $376^{\circ} \mathrm{C}$ 更熔点 $215-216^{\circ} \mathrm{C}$ 。

二、負离子基制备：各三联苯負离子基是椆三 联苯异构体与金属鉀作用制得。操作是在特制的硬 玻璃反应管內进行的。反应主管联接㣀空系統，主 管的一边为鉜鏡球，而另一边为細玻璃支管（队径

* 我們測得联萊實离子基为九組六綫。

** 紫外光譜系本所分析室胡振元及任华丽二同 志 湘 定。 